\title{
A NOTE ON THE CENTRAL LIMIT THEOREM FOR SQUARE-INTEGRABLE PROCESSES
}

\author{
MARJORIE G. HAHN
}

\begin{abstract}
A method is given for constructing sample-continuous processes which do not satisfy the central limit theorem in $C[0,1]$. Let $\{X(t): t \in[0,1]\}$ be a stochastic process. Using our method we characterize all possible nonnegative functions $f$ for which the condition

$$
E(X(t)-X(s))^{2}<f(|t-s|)
$$

alone is sufficient to imply that $X(t)$ satisfies the central limit theorem in $C[0,1]$.
\end{abstract}

1. Introduction. Let $C=C[0,1]$ denote the space of real-valued continuous functions on the unit interval. Let $\left\{X_{n}, n \geqslant 1\right\}$ be a sequence of independent $C$-valued random variables with the same distribution, $\mathcal{L}(X)$. Assume that they are defined on the same probability space $(\Omega, \mathscr{F}, \operatorname{Pr})$ and that for $t \in[0,1]$, $E X(t)=0$ and $E X^{2}(t)<\infty$. Let $Z_{n}=\left(X_{1}+\cdots+X_{n}\right) / \sqrt{n} . X$ or $\mathfrak{L}(X)$ is said to satisfy the central limit theorem (CLT) in $C$ if there exists a samplecontinuous Gaussian process $Z$ such that for one and hence all sequences $\left\{X_{i}\right\}$ as above, $\mathfrak{L}\left(Z_{n}\right) \rightarrow \mathfrak{L}(Z)$ weakly in $C$; i.e., for every bounded continuous real function $g$ on $C, E g\left(Z_{n}\right) \rightarrow E g(Z) . Z$ is called the limiting Gaussian process.

In this note we give a method for constructing sample-continuous processes which do not satisfy the CLT in C. A first application of this method appears in Hahn (1977).

Using this method we will show that when the only known information about a process $X(t)$ is of the form

(1.1) for some $\varepsilon>0$ and some nonnegative function $f$ on $[0,1]$ which is nondecreasing on $[0, \varepsilon]$,

$$
E(X(t)-X(s))^{2} \leqslant f(|t-s|), \quad|t-s| \leqslant \varepsilon,
$$

then the best possible sufficient condition for $X$ to satisfy the CLT in $C$ is

$$
\int_{0} y^{-3 / 2} \mathbf{f}^{1 / 2}(y) d y<\infty,
$$

where

Received by the editors October 8, 1976.

AMS (MOS) subject classifications (1970). Primary 60F05.

Key words and phrases. Central limit theorem, second-order processes, random Fourier series.

- American Mathematical Society 1977 


$$
f(s)= \begin{cases}\inf _{y>1} y^{2} f(s / y) & \text { if } s \in[0, \varepsilon] \\ f(s) & \text { if } s>\varepsilon .\end{cases}
$$

In Hahn and Klass (1977) it was shown that under assumption (1.1), the best possible condition for determining sample-continuity is (1.2).

2. Method for constructing sample-continuous processes which do not satisfy the CLT in $C$. Let $\{X(t), t \in[0,1]\}$ be a stochastic process on a probability space $(\Omega, \mathscr{F}, P)$ which prossesses the following properties:

$$
E X^{2}(t)<\infty \text { for all } t \in[0,1] \text {. }
$$

There is a set $A \in \mathcal{F}$ with $P(A)=\delta>0$ which contains a decreasing sequence of sets $A_{n} \in \mathcal{F}$ with $A \supset A_{1}, P\left(A_{n}\right)>0$ for all $n$ and $\lim _{n \rightarrow \infty} P\left(A_{n}\right)$ $=0$ such that

(2.2) for each $\omega \in A$ there is a nonempty subset $T(\omega) \subset[0,1]$ with the property that if $t \in T(\omega)$ then

$$
\lim _{s \downarrow t} X(s, \omega)=\lim _{r \uparrow t} X(r, \omega)= \pm \infty
$$

(2.3) for each $\omega \in A, X(t, \omega)$ is continuous on $[0,1] \sim T(\omega)$;

(2.4) for $\omega \in A^{c}, X(t, \omega)$ is continuous on $[0,1]$.

It is easy to see that such processes exist. A few examples when $\Omega=[0,1]$ with Lebesgue measure, $A=[0,1]$ and $T(\omega)=\omega$ are

$$
X(t, \omega)= \begin{cases}\text { either }|t-\omega|^{-1 / 4} \text { or } \log |t-\omega| & \text { if } t \neq \omega, \\ 0 & \text { if } t=\omega .\end{cases}
$$

A stochastic process $X$ with the above properties is not sample-continuous. However, as we will now show, it can be modified in such a way that it is both sample-continuous and does not satisfy the CLT in $C$.

We begin by choosing a function $R$ from $\Omega$ to $[0, \infty)$ for which

$$
\lim _{n \rightarrow \infty} n P\{\omega \in A: R(\omega) \geqslant \sqrt{n}\}=\infty .
$$

To see that such a function exists, let $A_{n}$ be the decreasing sequence of sets contained in $A$. Let $a_{n}=P\left(A_{n}\right)$. Extract a decreasing subsequence $a_{n_{k}}$ with the property that $k^{2} a_{n_{k-1}} \rightarrow \infty$. Let $R^{2}(\omega)=\inf \left\{k: \omega \notin A_{n_{k}}\right\}$. Then $\{\omega \in A$ : $\left.R^{2}(\omega) \geqslant k\right\}=A_{n_{k-1}}$, so $R(\omega)$ satisfies (2.5).

The desired modification of $X(t, \omega)$ is now obtained by first letting

$$
\tilde{X}(t, \omega)= \begin{cases}(\operatorname{sgn} X(t, \omega))(|X(t, \omega)| \wedge R(\omega)) & \text { if } t \notin T(\omega), \\ (\operatorname{sgn} X(t, \omega)) R(\omega) & \text { if } t \in T(\omega),\end{cases}
$$

and finally symmetrizing to yield

$$
Y(t, \omega)=Y(t, \omega \times k)= \begin{cases}\tilde{X}(t, \omega) & \text { if } k=0 \\ -\tilde{X}(t, \omega) & \text { if } k=1\end{cases}
$$


on the space $(\Omega \times\{0,1\}, P)$ where $P \equiv P \times\left(\delta_{0} / 2+\delta_{1} / 2\right)$.

THEOREM 1. The sample-continuous process $Y(t, \omega)$ does not satisfy the CLT in c.

Proof. Let $Y^{(i)}(t), i=1,2, \ldots$ denote i.i.d. copies of $Y(t), S_{n}(t)$ $=\sum_{i=1}^{n} Y^{(i)}(t)$ and $Z_{n}(t)=S_{n}(t) / \sqrt{n}$. We can assume that the independent copies of $Y$ are taken on a product space, $Y^{(i)}(t, \omega)=Y(t, \omega(i))$ where $\omega(i)=\omega(i) \times j, j=0$ or 1 .

In order to show that $Y(t)$ does not satisfy the CLT it suffices to show that $\left\{Z_{n}\right\}$ is not uniformly bounded in probability, i.e., there exists $\varepsilon>0$ such that for $b>0$ there is an $n(b)$ for which $\mathbf{P}\left\{\sup _{t}\left|Z_{m(b)}(t)\right| \geqslant b\right\}>\varepsilon$.

We begin by showing that for any $b>0$, there exists $N_{b}$ such that $n \geqslant N_{b}$ implies that

$$
\mathbf{P}\left\{\max _{1<i<n} \sup _{t}\left|Y^{(i)}(t)\right| / \sqrt{n}>2 b\right\}>\frac{1}{2} .
$$

Since $\sup _{t}\left|Y^{(i)}(t, \omega)\right| / \sqrt{n}=R(\omega(i)) / \sqrt{n}$,

$$
\begin{aligned}
\mathbf{P}\{\omega \in \Omega \times & \left.\{0,1\}: \max _{1<i<n} \sup _{t}\left|Y^{(i)}(t, \omega)\right| / \sqrt{n}<2 b\right\} \\
& =P^{n}\left\{\tilde{\omega} \in \Omega^{n}: \max _{1<i<n} R(\omega(i)) / \sqrt{n}<2 b\right\} \\
& =(P\{\omega \in \Omega: R(\omega) / \sqrt{n}<2 b\})^{n} \text { by independence } \\
& \leqslant \exp (-n P\{\omega \in \Omega: R(\omega) \geqslant 2 b \sqrt{n}\}) \rightarrow 0 \text { as } n \rightarrow \infty \text { by }(2.5) .
\end{aligned}
$$

Thus, there exists $N_{b}$ such that $n \geqslant N_{b}$ implies

$$
\mathbf{P}\left\{\max _{1<i<n} \sup _{t}\left|Y^{(i)}(t)\right| / \sqrt{n}<2 b\right\}<\frac{1}{2}
$$

and hence,

$$
\mathbf{P}\left\{\max _{1<i<n} \sup _{t}\left|Y^{(i)}(t)\right| / \sqrt{n} \geqslant 2 b\right\}>\frac{1}{2}
$$

Consequently, letting $\varepsilon=\frac{1}{4}$, if $n \geqslant N_{b}$,

$$
\mathbf{P}\left\{\max _{1<i<n} \sup _{t}\left|S_{i}(t)\right| / \sqrt{n} \geqslant b\right\}>2 \varepsilon .
$$

Applying the Lévy inequality for processes (see Dudley (1967), Lemma 4.4, p. 300 or Kahane (1968), Lemma 1, p. 12), we see that if $n \geqslant N_{b}$ then

$$
\mathbf{P}\left\{\sup _{t}\left|Z_{n}(t)\right| \geqslant b\right\} \geqslant \frac{1}{2} \mathbf{P}\left\{\max _{1<i<n} \sup _{t}\left|S_{i}(t)\right| / \sqrt{n} \geqslant b\right\}>\varepsilon
$$


3. Moment conditions on increments and the CLT. Let $\{X(t), t \in[0,1]\}$ be a stochastic process satisfying properties (1.1) and (1.2). As shown in Hahn and Klass (1977), proof of Theorem $1, E(X(t)-X(s))^{2} \leqslant 4 f(|t-s|)$. Consequently, by Theorem 2.5 of Hahn (1977), condition (1.2) is sufficient for $X$ to satisfy the CLT in C. The following theorem shows that this result is best possible.

THEOREM 2. If $f$ is a nonnegative function which is nondecreasing on $[0, \varepsilon]$ and such that $\int_{0} y^{-3 / 2} \mathbf{f}^{1 / 2}(y) d y=\infty$, there exists a sample-continuous process $Y(t, \omega)$ which does not satisfy the CLT in C but such that

$$
E(Y(t)-Y(s))^{2} \leqslant f(|t-s|), \quad|t-s| \leqslant \varepsilon .
$$

PRoof. In $\$ 4$ of Hahn and Klass (1976) a real-valued stochastic process, $X(t, \omega)$, was constructed on $[0,1] \times([0,1]$, Lebesgue) such that for each $t$,

$$
X(t, \omega)= \begin{cases}(2 \sqrt{2} \pi)^{-1} \sum_{k \geqslant 1} b_{k} \cos 2 \pi k(t-\omega), & 0<|t-\omega|<1, \\ 0 & |t-\omega|=0 \text { or } 1,\end{cases}
$$

where the sequence $\left\{b_{k}\right\}$ has the following properties:

(1) $b_{k} \geqslant b_{k+1}$

(2) $\sum_{k>1} b_{k}=\infty$;

(3) $\sum_{k=1}^{j} k^{2} b_{k}^{2}+j^{2} \sum_{k \geqslant j+1} b_{k}^{2} \leqslant j^{2} \mathrm{f}(1 / j)$;

(4) $k b_{k}$ is bounded.

For this process $E(X(t)-X(s))^{2} \leqslant f(|t-s|)$. Since the sequence $\left\{b_{k}\right\}$ decreases, $X(t, \omega)$ is continuous for $0<|t-\omega|<1$. As shown in Lemma 3 of Hahn and Klass, conditions (1), (2), and (4) imply that $\lim _{t \rightarrow \omega} X(t, \omega)=\infty$. Since $\cos 2 \pi k x=\cos 2 \pi k(1-x)$, the same argument shows that for fixed $\omega=0$, or $1, \lim _{|t-\omega| \rightarrow 1} X(t, \omega)=\infty$.

Let $R(\omega)=(1-\omega)^{-1} . R(\omega)$ satisfies (2.5). The desired sample-continuous process $Y(t, \omega)$ on $\left([0,1] \times\{0,1\}\right.$, Lebesgue $\left.\times\left(\delta_{0} / 2+\delta_{1} / 2\right) \equiv \lambda \times \mu\right)$ may be derived from $X(t, \omega)$ by the method given in $\$ 2$. Theorem 1 now shows that $Y$ does not satisfy the CLT in $C$.

Furthermore,

$$
\begin{aligned}
E_{\lambda \times \mu}(Y(t)-Y(s))^{2} & =E_{\lambda}(\tilde{X}(t)-\tilde{X}(u))^{2} \leqslant E(X(t)-X(s))^{2} \\
& \leqslant f(|t-s|), \quad|t-s| \leqslant \varepsilon . \quad \square
\end{aligned}
$$

\section{REFERENCES}

R. M. Dudley (1967), The sizes of compact subsets of Hilbert space and continuity of Guassian processes, J. Functional Analysis 1, 290-330.

M. G. Hahn (1977), Conditions for sample-continuity and the central limit theorem, Ann. Probability (to appear).

M. G. Hahn and M. J. Klass (1977), Sample-continuity of square-integrable processes, Ann. Probability (to appear).

J. P. Kahane (1968), Some random series of functions, Heath, Boston, Mass.

Department of Statistics, University of California, Berkeley, California 94720

Current address: Department of Mathematics, Tufts University, Medford, Massachusetts 02155 